\title{
Infancia y contenidos audiovisuales online en España: Una aproximación al consumo y a la mediación parental en las plataformas OTT
}

Childhood and online audiovisual content in Spain: An approach to consumption and parental mediation on OTT platforms

Infância e conteúdos audiovisuais online em Espanha: Uma abordagem ao consumo e mediação parental em plataformas OTT

María Marcos Ramos

Profesora Ayudante Doctora

(Universidad de Salamanca)

http://orcid.org/0000-0003-3764-7177

España

María de la Peña Mónica Pérez Alaejos

Profesora Contratada Doctora

(Universidad de Salamanca)

http://orcid.org/0000-0001-9022-9922

España
Marta Cerezo Prieto

Personal Investigador en Formación

(Universidad de Salamanca)

https://orcid.org/0000-0002-4620-8654

España

Marina Hernández Prieto

Profesora Ayudante Doctora

(Universidad de Salamanca)

http://orcid.org/0000-0001-9674-6883

España

Fecha de recepción: 9 de febrero de 2020

Fecha de revisión: 9 de febrero de 2020

Fecha de aceptación: 23 de abril de 2020

Fecha de publicación: 1 de julio de 2020

Para citar este artículo: Marcos Ramos, M., Pérez Alaejos, M. P. M, Cerezo Prieto, M. y Hernández Prieto. M. (2020). Infancia y contenidos audiovisuales online en España: Una aproximación al consumo y a la mediación parental en las plataformas 0TT, Icono 14, 18 (2), 245-268. doi: 10.7195/ri14.v18i2.1560 


\section{Resumen}

La convergencia multimedia y la llegada de Internet al mercado audiovisual ha afectado a las pautas de consumo y recepción de contenidos en el entorno familiar $y$ en los procesos de alfabetización mediática de los menores. A pesar de que las cifras de consumo de televisión lineal por parte de los más pequeños se mantienen, es innegable la penetración progresiva en los hogares españoles de las plataformas audiovisuales, siendo fundamental el papel que realizan los padres/tutores.

El objetivo de esta investigación es identificar las prácticas de uso en dichas plataformas por parte de niños/as de infantil y primaria en España, y analizar los sistemas de mediación parental en el nuevo escenario de convergencia mediática, a partir de un estudio descriptivo realizado con 431 padres/madres o tutores. Para llevarlo a cabo se ha utilizado la herramienta metodológica Qualtrics, mediante la aplicación de un cuestionario a través de un muestreo en bola de nieve.

Tras el análisis de los resultados se constatan cambios importantes en la mediación parental, acordes con el incremento progresivo en la edad de los niños/as, aunque la regulación más frecuente en ambas franjas de edad está vinculada al tiempo que los progenitores o tutores permiten a los menores pasar frente a los diferentes dispositivos, más que en las restricciones en torno a los contenidos. Además, no se constatan diferencias significativas respecto al tipo de productos audiovisuales que consumen en la televisión lineal y en las plataformas OTT, predominando las series y las películas infantiles en las edades más tempranas.

Palabras clave: Mediación parental; Niños; Consumo audiovisual; Televisión; Alfabetización mediática

\section{Abstract}

Multimedia convergence and the arrival of the Internet in the audiovisual market have affected the patterns of consumption and reception of content in the family environment and in the media literacy processes of minors. Despite the fact that the figures for linear television consumption by the youngest children remain unchanged, the progressive penetration of audiovisual platforms in Spanish households is undeniable, and the role played by parents/guardians is fundamental. 
The aim of this research is to identify the practices of use in these platforms by children of infant and primary school in Spain, and to analyse the parental mediation systems in the new scenario of media convergence, based on a descriptive study carried out with 431 parents or tutors. To carry it out, the Qualtrics methodological tool has been used, by means of the application of a questionnaire through a snowball sampling.

After the analysis of the results, important changes were observed in parental mediation, in accordance with the progressive increase in the age of the children, although the most frequent regulation in both age groups is linked to the time that the parents or guardians allow the children to pass through the different devices, rather than in the restrictions around the contents. Moreover, there are no significant differences in the type of audiovisual products they consume on linear television and OTT platforms, with children's series and films predominating at the earliest ages.

Key Words: Parental mediation; Children; Audiovisual consumption; Television; Media literacy

\section{Resumo}

A convergência multimídia e a chegada da Internet ao mercado audiovisual afetaram os padrões de consumo e recepção de conteúdo no ambiente familiar e nos processos de alfabetização midiática de menores. Embora os valores para o consumo linear de televisão sejam menores, a penetração progressiva nos lares espanhóis das plataformas audiovisuais é inegável, sendo fundamental o papel desempenhado pelos pais / responsáveis.

0 objetivo desta pesquisa é identificar as práticas de uso nessas plataformas por filhos de filhos primários e secundários da Espanha e analisar os sistemas de mediação parental no novo cenário de convergência da mídia, a partir de um estudo descritivo realizado com 431 pais ou mães ou responsáveis. Para isso, foi utilizada a ferramenta metodológica Qualtrics, através da aplicação de um questionário através de uma amostra de bola de neve.

Após a análise dos resultados, mudanças importantes na mediação dos pais são observadas, em consonância com o aumento progressivo da idade das crianças, embora a regulação mais frequente nas duas faixas etárias esteja ligada ao tempo que os pais ou responsáveis permitem os menores passam na frente dos diferentes dispositivos, mais do que nas restrições em torno do conteúdo. Além disso, não há 
MONOGRÁFICO

diferenças significativas em relação ao tipo de produtos audiovisuais consumidos nas plataformas de televisão linear e OTT, prevalecendo as séries e os filmes infantis nas idades mais precoces.

Palavras chave: Mediação parental; Filhos; Consumo audiovisual; Televisão; Alfabetização midiática

\section{Introducción}

Tan importante es investigar qué contenidos audiovisuales consumen los más pequeños, cómo el control que se ejerce en sus entornos sobre el acceso a estos. Si con la televisión tradicional la mediación parental resultó ser una herramienta fundamental para ayudar al niño a poder desentrañar mensajes mediáticos (Livingstone, 2007), en el panorama actual, caracterizado por la abundancia y por la diversidad, esta se torna en fundamental. No se debe olvidar que la televisión, ya sea vista por cable o través de plataformas online, es uno de los mayores agentes socializadores siendo la mediación familiar imprescindible (Torrecillas-Lacave, 2013). Una mediación que puede ser entendida como una "instancia cultural desde donde el público de los medios produce y se apropia del significado y del sentido del proceso comunicativo" (Martín-Barbero, 1987) o como un "proceso estructurante que configura y reconfigura tanto la interacción de los miembros de la audiencia con la televisión como la creación por ellos del sentido de esa interacción" (Orozco, 1996, p. 74). Se ha demostrado que la mediación familiar resulta fundamental (Gabelas y Marta-Lazo, 2008) al ser la familia "el grupo natural para ver la televisión" (0rozco, 1996, p. 41) y al ser "uno de los factores más determinantes en la relación que el niño establezca con la pequeña pantalla, tanto en lo que respecta a los tiempos y dietas de consumo, como en los modos de visionado de los contenidos y los posibles beneficios o perjuicios cognitivos y morales que deriven de esa relación" (Del Río, Álvarez, Del Río, 2004, p. 286) ya que "los niños que mejor parados salen de su relación con la televisión son aquellos cuyos padres despliegan más estrategias de interacción-triangulación con sus hijos y la pantalla televisiva" (Del Río, Álvarez, Del Río, 2004, p. 286), tal y como demuestran recientes investigaciones como la de Gabelas y Marta-Lazaro (2008).

Se han señalado diferentes formas de mediación familiar, como la integración dentro de los hogares, la organización del tiempo y del espacio, la regulación (Barrios, 1992) 
o la crítica y el juicio a los contenidos (Aguaded, 1999). Tal y como señala Aguaded es fundamental que entre los padres y los hijos se establezca un diálogo que contextualice lo que se está viendo pues "no basta con que el niño no esté sólo físicamente ante la pantalla. Es preciso que no se sienta sólo como telespectador, que comparta la experiencia, que pueda dialogar, confrontar" (Ferrés, 1994, p. 137). Aguaded sugiere "la necesidad de establecer estrategias de atenuación, en las que la información, el diálogo y las opciones se vuelvan facetas básicas para contrarrestar lo que denomina el poder de la caja mágica" (Aguaded,1999, p. 36). Cuando los padres optan por utilizar el diálogo pueden seguir diferentes estrategias, como patrones de mediación positiva, negativa, instructiva o restrictiva (Austin, 2001). En una investigación pionera, Abelman y Pettey (1989) señalaron tres tipos de mediación parental: restrictiva; la evaluativa; y, por último, la desfocalizada. Van der Voort, Nikken y Van Lil (1992) indican que la mediación puede ser restringida, orientadora y desenfocada. Pindado (1998) califica a estos tres modelos como TV-peligro, TV-«está ahí» y TV-niñera. Urra (1998) utiliza otro término similar, "canguro catódico" y Llopis (2004), tras realizar un análisis factorial y de conglomerados, indica que las familias españolas pueden clasificarse en función de tres estrategias de mediación: controladoras-restrictivas, permisivas y orientadoras. Torrecillas-Lacave (2013) clasifica en cuatro tipos la mediación efectiva: autocontrol, heterocontrol, control y descontrol. Vílchez-Martín (1999) indica que hay cuatro tipos de estilo mediación de corte cualitativo: didáctico, rigorista, cómodo y paternal.

Realizar una mediación en los hogares, aunque no sea intensiva ni severa, debería ser cuanto menos una práctica habitual, sin embargo, diversas investigaciones han demostrado que prima "el desinterés o permisividad de algunos padres por el indiscriminado consumo televisivo de sus hijos" (Aparici, 1994, p. 1) ya que la mayor parte de las veces los niños ven la televisión solos (Aguaded y Díaz-Gómez, 2008), tal y como demuestran las cifras aportadas por López Romero y Monedero Morales ya que "del $65,8 \%$ de alumnos que consumen televisión junto a familiares, el $56,4 \%$ lo hacen con los padres. El resto del consumo televisivo lo realizan bien a solas, con amigos, con hermanos y en menor porcentaje con los abuelos" (Romero y Monedero 2008, p. 271).

Otras investigaciones indican la ausencia de mandatos (García-Cortázar et al. 1998) o falta de coherencia entre lo que los niños deberían ver y lo que ven (VílchezMartín, 1999). Es lo que Núñez-Ladevéze y Pérez-Ornia denominan disonancia prag- 
MONOGRÁFICO

mática: "se trata de contrastar entre los criterios normativos que los padres expresan acerca de cómo deben controlar lo que ven sus hijos y sus ideas acerca de lo que no conviene que vean y la conducta efectiva" (Ladevéze y Pérez-Ornia, 2002, p 139).

Torrecillas-Lacave (2013) indica que deben ser analizados los siguientes contextos de recepción televisiva: escenario de consumo, escenario situacional, mediación familiar, comportamiento efectivo de los hijos y características de los productos consumidos por los niños.

Han sido numerosos los estudios que se han dedicado a analizar cómo era la mediación parental que se hacía en los hogares con la denominada televisión tradicional (Abelman y Pettey, 1989; Van der Voort, Nikken y Van Lil, 1992; Barrios, 1992; Ferrés, 1994, 2005; Aguaded, 1995, 1997, 1999, 2005; Pindado, 1998; Austin, 2001; Del Río, Álvarez, Del Río, 2004; Fernández, Domínguez, Revilla y Anagnostou, 2004; Gabelas y Marta-Lazo, 2008; Aguaded y Díaz-Gómez, 2008; López Romero y Monedero Morales, 2008; Torrecillas-Lacave, 2013, etc.). Por el contrario, son muchos menos los estudios que han analizado cómo es esa mediación parental con los contenidos que les llegan vía Internet (Solomon-Moore et al., 2018; Hiniker et al., 2016).

En este artículo se presentan los resultados obtenidos, a partir de un estudio cuantitativo para el que se ha utilizado la herramienta metodológica Qualtrics, y en el que se ha analizado el consumo de los contenidos audiovisuales en las plataformas 0TT y la mediación familiar que se realiza. Para ello se han analizado las respuestas de los 431 padres, madres y tutores de niños/as entre 3 y 12 años que han participado en este estudio.

\section{Metodología}

Para realizar esta investigación se ha realizado un estudio exploratorio en el que se ha realizado una encuesta a 431 personas que debían autocumplimentar un cuestionario realizado en Qualtrics que estaba compuesto por 145 preguntas con las que se podían analizar 637 variables diferentes. El cuestionario podía ser completados por padres/madres/tutores de niños/as de entre 3 y 12 años. 
MONOGRÁFICO

\subsection{Análisis de la muestra}

La muestra es de 431 sujetos repartidos en dos grupos: 168 se corresponden con padres, madres o tutores con hijos/as entre 3 y 6 años y 263 de hijos/as entre 7 y 12 años. En el primer intervalo el $54,2 \%$ son varones frente al $45,8 \%$ de las niñas, mientras que en segundo intervalo la cifra de niños aumenta hasta el 55,1\% frente al 44,9\% de las niñas. En cuanto a la edad, la media se sitúa en 9,73 (DT=2,93) y por intervalos es la siguiente: $4,62(\mathrm{DT}=1,09)$ en el primero y 7,72 $(\mathrm{DT}=1,78)$ en el segundo.

Fijándonos en la profesión de los progenitores, el 70,8\% declara tener un trabajo a tiempo completo, el 19,7\% son empleados a tiempo parcial, el 8,6\% han indicado que estaban en el paro $y$, finalmente, el $0,7 \%$ han indicado que son jubilados. Respecto al estado civil, el porcentaje mayoritario en ambos intervalos declaran estar casados, el 70,02\% y el 78,3\% respectivamente. En lo que respecta al nivel de estudios de los progenitores el $62,1 \%$ indicó que tiene estudios universitarios superiores en el primer intervalo y el $60,1 \%$ en la segunda franja de edad. La media de hijos que tienen los encuestados es de 1,84 (DT=0,85).

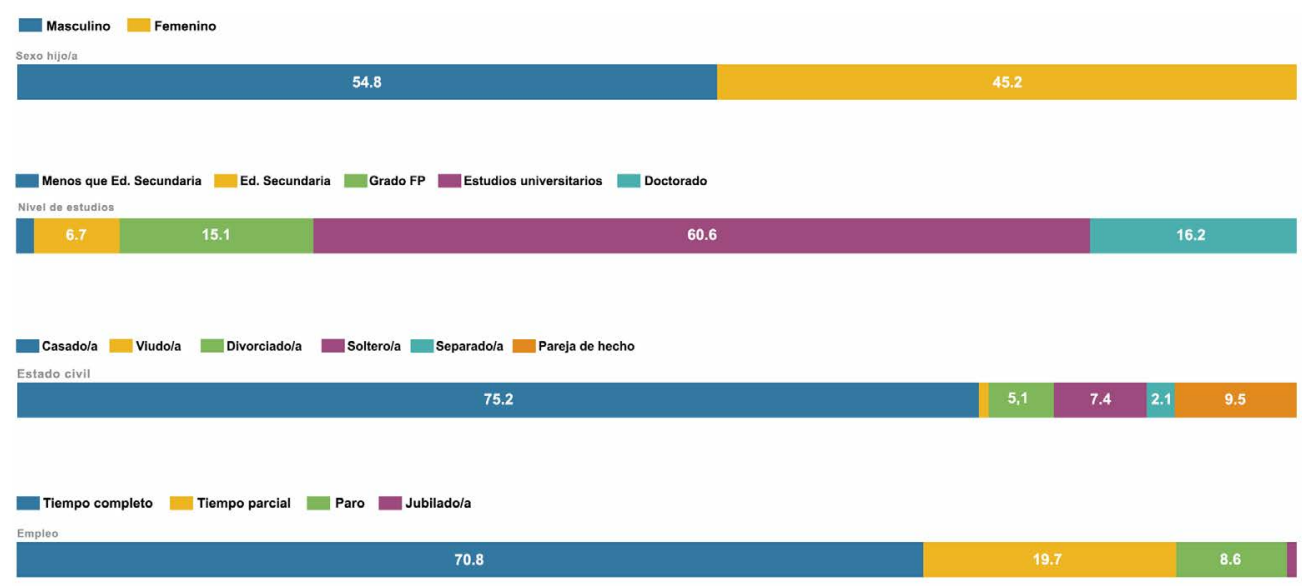

Gráfico 1: Características sociodemográficas de la muestra.

Fuente: elaboración propia.

También se consideró relevante analizar la conexión a Internet de los hogares. Así, todos declararon que tienen o utilizan algún tipo de conexión a Internet y 
MONOGRÁFICO

tan sólo 18 de ellos, lo que supone tan solo un 4,17\% no dispone de conexión en casa. Habría que resaltar que en el momento de realizar la encuesta, a pesar de no ser una tecnología plenamente implantada, el 3,94\% ya cuentan con una conexión 5G. El gasto medio mensual en contenidos audiovisuales online es entre 1 y $15 €$ y el modo en el que lo consumen se puede ver en la Tabla 1.

\begin{tabular}{|l|c|c|c|c|c|}
\hline & $\begin{array}{c}\text { Plataformas } \\
\text { de pago }\end{array}$ & $\begin{array}{c}\text { Plataformas } \\
\text { gratuitas }\end{array}$ & $\begin{array}{c}\text { Alquiler 0 } \\
\text { compra }\end{array}$ & Descargas & Otros \\
\hline $\begin{array}{l}\text { Intervalo 1 } \\
\text { (3-6 años) }\end{array}$ & $56 \%$ & $79,8 \%$ & $4,8 \%$ & $19,6 \%$ & $7,1 \%$ \\
\hline $\begin{array}{l}\text { Intervalo 2 } \\
\text { (7-12) }\end{array}$ & $56,7 \%$ & $84 \%$ & $4,6 \%$ & $24 \%$ & $4,6 \%$ \\
\hline
\end{tabular}

Tabla 1: Conexión a Internet. Fuente: elaboración propia.

Los padres con niños entre 3 y 6 años mencionaron además, otras plataformas que usaban con regularidad como Youtube Kids, Filmin o un programa de su centro escolar llamado Progrentis. Los participantes correspondientes a las edades entre 7 y 12 años manifestaron usar también Google Earth, Orange TV o Spotify.

\subsection{Objetivos y preguntas de investigación}

El presente trabajo pretende analizar si con la llegada de las plataformas de distribución de contenidos audiovisuales online han cambiado los usos y costumbres en el consumo de dichos contenidos por parte de los más pequeños y si la mediación parental ha variado respecto a lo que indicaban estudios precedentes basados en la televisión convencional.

Se pretende, por un lado, identificar las prácticas de uso de las plataformas de distribución de contenidos audiovisuales por parte de los niños en España y, además, identificar los contextos de recepción televisiva infantil en las 0TT, fijándonos en aspectos como:

a. Los escenarios de consumo.

b. El escenario situacional. 
c. El comportamiento efectivo.

d. Las características de los productos consumidos (Torrecillas-Lacave, 2013).

Del mismo modo, se busca identificar las diferencias en las variables más relevantes entre los dos grupos de edad establecidos en la muestra (infantil y primaria) para tratar de describir los cambios esenciales con el incremento progresivo de la edad, si los hubiese.

\section{Resultados}

Para poder identificar las prácticas de uso a través de las cuáles se consumen más contenidos audiovisuales por parte de los niños, se han analizado cuáles son las plataformas 0TT a la que están suscritos los encuestados, además de analizar cuáles son las que más ven y las mejor valoradas en cuanto a contenidos específicos para el público más joven.

Movistar es el operador de telefonía e Internet que predomina en la muestra, ya que 149 de los 431 hogares está abonado a dicha compañía, lo que supone un $34,57 \%$. Le siguen Vodafone con un $21,57 \%$ y Orange con $12,06 \%$ de abonados. Por el contrario, Amena es la compañía menos representada ya que solamente es utilizada en 2 hogares.

También se quiso saber qué plataformas audiovisuales ven con mayor frecuencia y se les pidió, además, que puntuarán cada plataforma o app en función de lo adecuada que la consideraran para el consumo infantil, tal y como puede verse en la tabla 2. Así, en el primer intervalo de edad, la que más ven es Clan TV que además es también la que los padres, madres o tutores consideran más apropiada para su consumo. Por el contrario, la considerada menos apropiada para la edad entre 3 y 6 años sería Youtube que, sin embargo, es vista por el 49,4\% de los niños de la muestra, cifra que aumenta en el segundo intervalo de edad siendo la más vista, con un $69,2 \%$. A pesar de ser la más vista, ningún padre/madre la considera la más adecuada. Sí que consideran la más apropiada Clan TV, los padres de los niños entre 7 y 12 años, que es vista en un 37,6\% de los casos correspondientes a esta franja de edad. Por el contrario, la calificada como menos apropiada es Amazon Video. 
MONOGRÁFICO

\begin{tabular}{|l|c|c|c|c|c|c|}
\hline & \multicolumn{3}{|c|}{ Intervalo 1 (3-6 años) } & \multicolumn{3}{c|}{ Intervalo 2(7-12) } \\
\hline & Más vistas & $\begin{array}{c}\text { Muy } \\
\text { apropiada }\end{array}$ & $\begin{array}{c}\text { Nada } \\
\text { apropiada }\end{array}$ & $(7-12)$ & $\begin{array}{c}\text { Muy } \\
\text { apropiada }\end{array}$ & $\begin{array}{c}\text { Nada } \\
\text { apropiada }\end{array}$ \\
\hline Netflix & $36,9 \%$ & $3,6 \%$ & $7,7 \%$ & $38,8 \%$ & $3,4 \%$ & $6,8 \%$ \\
\hline Movistar & $19 \%$ & $1,8 \%$ & $4,8 \%$ & $24,7 \%$ & $3,4 \%$ & $4,9 \%$ \\
\hline Movistar junior & $11,9 \%$ & $8,9 \%$ & $1,8 \%$ & $6,5 \%$ & $7,2 \%$ & $3,4 \%$ \\
\hline HB0 & $10,7 \%$ & $0,6 \%$ & $7,7 \%$ & $14,4 \%$ & $0,8 \%$ & $7,2 \%$ \\
\hline Amazon Video & $17,3 \%$ & $1,8 \%$ & $10,1 \%$ & $17,1 \%$ & $1,5 \%$ & $9,1 \%$ \\
\hline Filmin & $1.8 \%$ & $0,6 \%$ & $7,1 \%$ & $1,1 \%$ & $1,1 \%$ & $6,8 \%$ \\
\hline Youtube & $49,4 \%$ & $1,8 \%$ & $13,7 \%$ & $69,2 \%$ & $0,00 \%$ & $6,1 \%$ \\
\hline Youtube Kids & $45,2 \%$ & $8,3 \%$ & $1,8 \%$ & $26,6 \%$ & $9,9 \%$ & $2,3 \%$ \\
\hline Clan TV & $56,5 \%$ & $25 \%$ & $1,2 \%$ & $37,6 \%$ & $30,4 \%$ & $0,8 \%$ \\
\hline $\begin{array}{l}\text { No consume contenidos } \\
\text { audiovisuales de ninguna } \\
\text { plataforma online o app }\end{array}$ & $3,6 \%$ & & & $6,5 \%$ & & \\
\hline Otros & $6,5 \%$ & & & $5,7 \%$ & & \\
\hline T0TAL & 168 & 168 & 168 & 663 & $\mathbf{2 6 3}$ & $\mathbf{2 6 3}$ \\
\hline
\end{tabular}

Tabla 2: Consumo y valoración de las plataformas de contenidos audiovisuales. Fuente: elaboración propia.

Se midió también, utilizando un análisis de T de Student para muestras independientes, si el nivel de estudios de los padres se relacionaba con las plataformas que consumían sus hijos. Así, se contrastó que a mayor nivel de estudios de los progenitores las plataformas más consumidas son HBO $([429, N=431]=.934, p$ $<.05)$ y Filmin $(\chi 2[429, N=431]=.206, p<.005)$. Tendencialmente se encontró diferencias significativas en el caso de Amazon Prime $([429, N=431]=.137, p=$ .08). No se encontró relación estadísticamente significativa en las siguientes plataformas: Movistar, Netflix, Youtube, Movistar Junior, Youtube Kids, Clan TV.

Se midió en cada una de las opciones que proporcionaba la encuesta si existía una relación entre el nivel de estudios de los padres y las formas de consumo audiovisual de los niños/as (plataformas de pago, gratuitas, alquiler o compra y descarga). No se encontraron diferencias significativas, sin embargo, sí aparecieron esas diferencias significativas cuando se midió la forma de consumo de forma conjunta creando un índice agregado que aglutinaba todas las formas de consumo. Se detectó que había diferencias significativas 
entre el nivel de estudios y las formas de consumo de tal manera que a mayor nivel de estudios se empleaban más formas de consumo $([N=431]=.093, p<.05)$.

Para identificar los contextos de recepción televisiva infantil en las plataformas audiovisuales, primero se identificaron los escenarios de consumo, es decir, las características materiales del espacio en el que se produce la relación entre el niño y el contenido audiovisual. Dado que los contenidos audiovisuales no son solo consumidos en la actualidad a través del televisor, se amplió esta identificación a cualquier otro dispositivo mediante el cual se pudiesen consumir contenidos audiovisuales, como ordenadores, tablets, teléfonos móviles, etc. En ambos intervalos de edad el teléfono móvil es el dispositivo más usado en los hogares españoles, seguido del portátil entre los niños de 3 a 6 años y de la tablet o ipad entre los de 7 a 12 años (Gráfico 2).

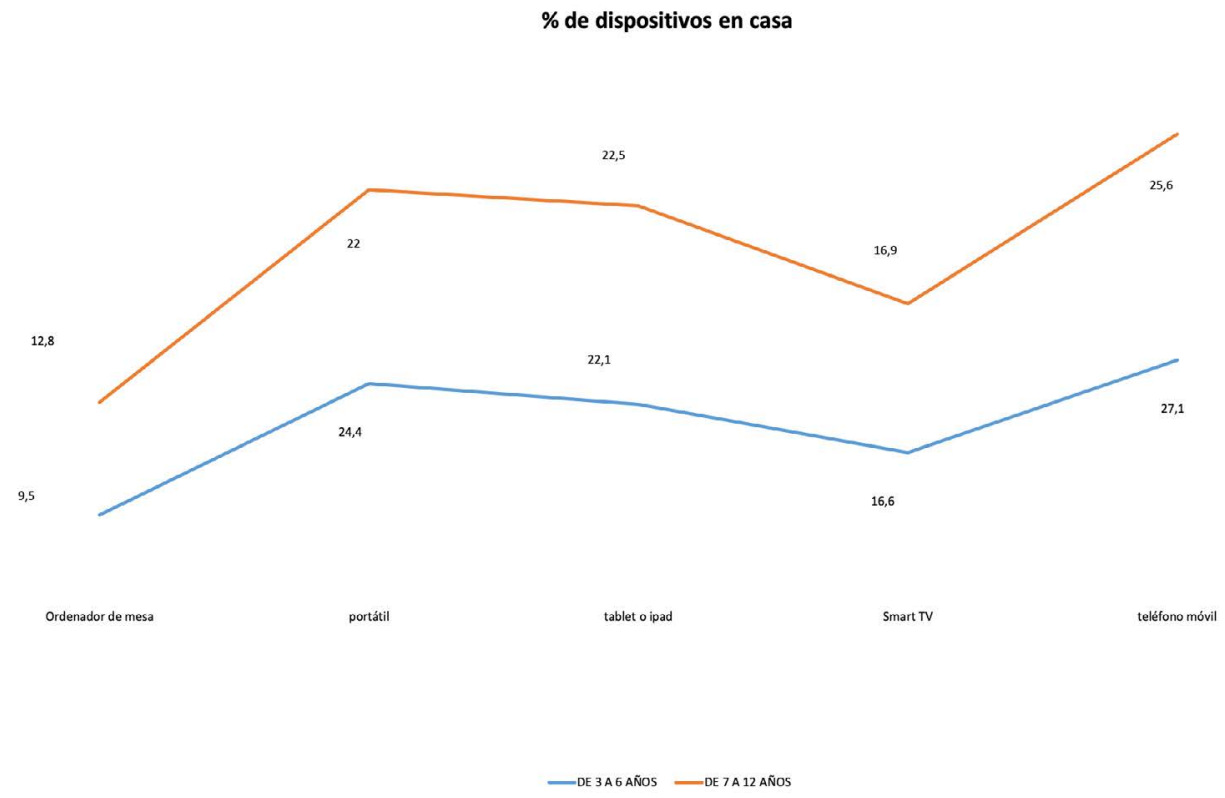

Gráfico 2: Dispositivos en los hogares.

Fuente: elaboración propia.

Si se analizan los dispositivos que los hijos utilizan para su propio consumo se pueden observar diferencias significativas entre los dos grupos de edad. En el primer intervalo, la respuesta mayoritaria es que el niño no posee ningún dispositivo para uso 


\section{MONOGRÁFICO}

propio, siendo esta respuesta considerablemente más baja en el del segundo intervalo. La tablet es el dispositivo que más usan los niños de entre 3 y 6 años, siendo también su uso mayoritario en el segundo grupo de edad. En el caso del teléfono móvil para uso personal también existen diferencias significativas, tan sólo el 1,1\% de los niños más pequeños disponen de él, mientras que el $19,2 \%$ de los casos de la franja de mayor edad declaran usarlo de manera personal para el consumo audiovisual.

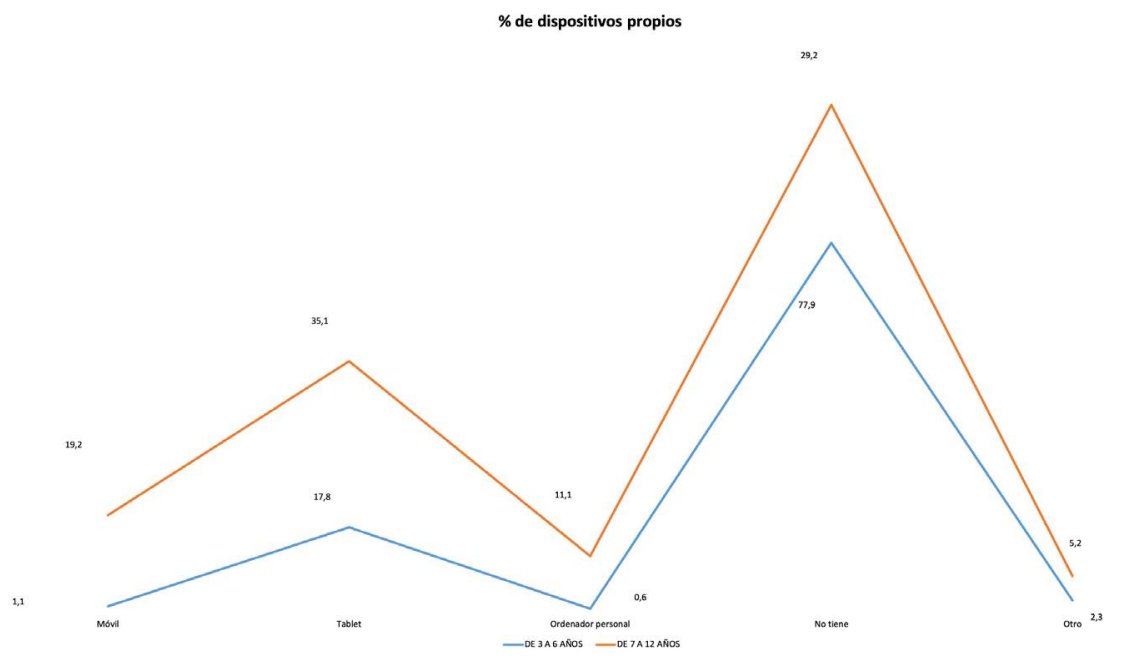

Gráfico 3: Dispositivos que tienen los niños/as.

Fuente: elaboración propia.

Para describir las diferentes situaciones en las que se produce el proceso de consumo audiovisual de los hijos, además de para identificar si se realiza una mediación parental y de qué tipo, resultaba fundamental analizar si los niños/as consumen contenidos audiovisuales en plataformas solos o acompañados. Así, si se analizan los datos en conjunto el $61,9 \%$ de los encuestados manifestó dejarles solos a veces, seguida de un $31,6 \%$ que contestó que no y, finalmente, la respuesta de sí seleccionada por un $6,5 \%$ de los casos. Si se profundiza en el dato en función de los dos intervalos de edad, en ambos la opción mayoritaria es a veces, siendo más frecuente en el grupo con más años. En el primer intervalo resulta significativa la respuesta de no, muy superior porcentualmente que en segundo grupo, en el que a los niños se les deja ver contenidos audiovisuales solos en mayor medida que en el intervalo anterior, donde los niños son más pequeños, tal y como muestra la tabla 3. 


\begin{tabular}{|l|c|c|c|}
\hline & $\begin{array}{c}\text { 3 a 6 } \\
\text { años }\end{array}$ & $\begin{array}{c}\text { 7 a 12 } \\
\text { años }\end{array}$ & Total \\
\hline Sí & $4,2 \%$ & $8 \%$ & $6,5 \%$ \\
\hline No & $47 \%$ & $21,7 \%$ & $31,6 \%$ \\
\hline A veces & $48,8 \%$ & $70,3 \%$ & $61,9 \%$ \\
\hline Total (N) & 168 & 263 & 431 \\
\hline
\end{tabular}

Tabla 3: ¿Consumen los niños contenidos audiovisuales solos? Fuente: elaboración propia.

Se les preguntó también si se regulaba el consumo de contenidos audiovisuales de sus hijos y cómo establecían esa regulación siendo en ambos casos la respuesta mayoritaria la regulación mediante limitaciones temporales.

\begin{tabular}{|l|c|c|c|}
\hline & $\begin{array}{c}\text { 3 a 6 } \\
\text { años }\end{array}$ & $\begin{array}{c}\mathbf{7} \text { a 12 } \\
\text { años }\end{array}$ & Total \\
\hline Limitaciones temporales & $59,5 \%$ & $54,4 \%$ & $6,5 \%$ \\
\hline Limitaciones de contenido & $25,6 \%$ & $25,9 \%$ & $31,6 \%$ \\
\hline Como premio & $3 \%$ & $7,6 \%$ & $61,9 \%$ \\
\hline No se regula. Lo puede ver el tiempo que el niño/a quiera & $1,2 \%$ & $1,5 \%$ & 431 \\
\hline Otros & $10,7 \%$ & $10,6 \%$ & \\
\hline Total (N) & 168 & 263 & \\
\hline
\end{tabular}

Tabla 4: Tipos de regulación de contenidos audiovisuales. Fuente: elaboración propia.

En el primer intervalo se indicaron además otras formas de regulación más esporádicas y en respuesta abierta como "mientras cena" o por "mal comportamiento". Son destacables respuestas como "si se porta mal no ve la televisión", "hablando con ellos y explicando los límites" o "Youtube y youtube kids no está instalado en su dispositivo personal, tan solo le permitimos esta app en nuestro teléfono y fuera de casa como entretenimiento". En el segundo intervalo de edad, las respuestas a esta pregunta fueron las siguientes: "con mamá supervisando", "de momento no necesita regulación porque lo pide muy esporádicamente y ella misma lo deja enseguida", "si no hay buen comportamiento no se ve tele, móvil, etc.", "vemos solo cine los viernes en la tele" o "audiovisuales no son para mi hija una opción de entretenimiento", "suele leer pintar o salir a la calle para entretenerse" o "como buenamente puedo".

Por otro lado, se analizó también si se tenían instalados mecanismos de control parental y en el caso de que los tuvieran cuáles eran los que usaban en mayor medida. 
MONOGRÁFICO

Así, los padres de los más pequeños contestaron que no en un 53\% de los casos, seguida de sí con 43,5\% y un 3,6\% no sabían si disponían de él. Sobre cuáles eran los que usaban indicaron los siguientes: límite de tiempo y contraseña, inhabilitación de las descargas, Google family, programa de vigilancia, pin parental, el propio de la televisión que bloquea contenidos destinados a mayores de 7 años, el antivirus Karpesky, el filtro de Netflix, etc. Entre los padres de los niños de primaria, la respuesta mayoritaria fue la de que sí había instalados controles parentales en un 52,9\%, seguida por no por un $44,5 \%$ y no sé fue elegida en un $2,7 \%$ de los casos. En este intervalo las respuestas abiertas fueron más numerosas y destacan las siguientes: los programas Qstodio, Family link, Google family, Control Parental Guidance, Parental click o Sophos, los filtros y bloqueos que poseen las propias aplicaciones o la utilización de la aplicación Find my kids para controlar el tiempo de consumo.

Se consideró interesante saber si el contenido consumido por los niños/as era revisado por los progenitores con anterioridad al visionado. Si nos fijamos en los datos de los/as niños/as de infantil, la respuesta mayoritaria fue la de "siempre" con un 50\%, seguida de "no siempre" con un 35,7\% y "no" con un 14,3\%. En el grupo de padres de niños/as de primaria, la respuesta mayoritaria fue la de "no siempre" seleccionada por un 49,4\%, seguida de "sí" un 36,5\% y "no" con un 14,1\%.

De manera complementaria se les preguntó de dónde obtenían información sobre los contenidos que veían sus hijos/as y se les pidió que indicaran todas las opciones que utilizasen, siendo el grupo de amigos el que ejerce en mayor medida como prescriptor.

\begin{tabular}{|l|c|c|}
\hline & 3 a 6 años & $\mathbf{7}$ a 12 años \\
\hline Recomendaciones de amigos & $48,2 \%$ & $49,4 \%$ \\
\hline Redes sociales & $14,9 \%$ & $24,7 \%$ \\
\hline Webs especializadas & $31 \%$ & $31,2 \%$ \\
\hline No me informo & $17,3 \%$ & $16,3 \%$ \\
\hline Otros & $20,8 \%$ & $23,2 \%$ \\
\hline N total de respuestas & 168 & 263 \\
\hline
\end{tabular}

Tabla 5: ¿De dónde se obtiene la información de los contenidos audiovisuales? Fuente: elaboración propia. 
En cuanto a las respuestas abiertas, merece la pena desatacar las siguientes en el primer intervalo: "contenidos que ve en clase, series que ya ha visto o he visto yo de pequeña", "tengo en cuenta la edad a la que van dirigidos y si no son para su edad no permito que lo vean y así se lo explico" o "nos lo proponen en el colegio". En el segundo intervalo de edad las respuestas han sido cuantitativamente más numerosas: "yo misma reviso su móvil/ ordenador y visualizo lo que considero nuevo", "cuando quiere ver algo me pregunta primero si puede ver ese contenido en concreto, yo busco información y si me parece correcto le dejo verlo", "revistas de papel o artículos de prensa" o "recomendación de los profesores o de aplicaciones como Google classroom".

Se midió también si existían diferencias significativas entre el nivel de estudios de los padres/madres/tutores y algunas cuestiones relevantes relacionadas con el consumo de los niños/as. Así, se encontraron diferencias significativas con el control parental $([N=431]=4.125, p<.05)$ y con la regulación o con la revisión del contenido anterior al visionado por parte de los padres $([N=431]=-.095, p<.05)$. Merece la pena destacar que a mayor nivel de estudios de los progenitores el consumo de contenidos audiovisuales se regula en mayor medida de las siguientes maneras: como premio, con limitaciones temporales o con limitaciones de contenido. También resulta significativa la relación entre nivel de estudios con las formas en las que los padres obtienen la información sobre los contenidos, en la que solo la respuesta de "redes sociales" arrojó una relación significativa $([N=431]=.182, p<.001)$, dado que, son las personas con menor nivel de estudios las que más se informan por redes sociales.

Para la cuestión que abordaba los cambios en las formas de consumo de contenidos audiovisuales según el soporte (tv tradicional versus OTT) se consideró necesario saber el número de horas que los niños/as dedican a ver contenidos audiovisuales, tanto en soporte convencional como en plataformas online. Una de las primeras premisas era identificar si los niños/as siguen consumiendo televisión tradicional y los datos muestran que sí. En el primer intervalo, consumen televisión convencional el $64,3 \%$ y en el segundo, el porcentaje aumentaba hasta el $76 \%$.

En la tabla 6 se recogen los datos facilitados sobre el número de horas que consumen sus hijos contenidos audiovisuales en televisión convencional y en plataformas, distinguiendo además si lo hacen entre semana, fin de semana o en periodos vacacionales. 
MONOGRÁFICO

Si se analizan las horas máximas que consumen los niños/as entre semana, fin de semana o periodos vacacionales llama la atención la cantidad. Así, en el intervalo de niños de 3 a 6 años, se ha indicado que el consumo máximo entre semana es de 10 horas, en el fin de semana sube a 12 y en períodos vacacionales suman más de 20 horas semanales, lo que significa que se ven casi tres horas diarias de televisión. En el intervalo de 7 a 12 años, las cifras cambian a 10, 15 y más de 20 horas.

En cuanto al consumo de contenidos en plataformas llama especialmente la atención las horas máximas de consumo en algunos casos, como las 15 horas de consumo entre semana en el período infantil, cifra que se dispara a 25 entre los niños/as de 7 a 12 años. En los fines de semana, las horas máximas de consumo son 10 horas en los niños de 3 a 6 años y 15 horas en los niños/as de primaria. Son más significativas las cifras de los períodos vacacionales ya que consumen más de 20 horas en los dos intervalos de edad, sin embargo, es más reveladora la cantidad de respuestas recogidas en cifras más altas entre los niños de 7 a 12 años, pues el $6,25 \%$ de la muestra ven más de 20 horas semanales en períodos vacacionales.

\begin{tabular}{|l|c|c|c|c|c|c|}
\hline & \multicolumn{3}{|c|}{ CONVENCIONAL } & \multicolumn{3}{c|}{ PLATAFORMAS } \\
\hline & $\begin{array}{c}\text { Entre } \\
\text { semana }\end{array}$ & $\begin{array}{c}\text { Fin de } \\
\text { semana }\end{array}$ & $\begin{array}{c}\text { Vacacional } \\
\text { semanal }\end{array}$ & $\begin{array}{c}\text { Entre } \\
\text { semana }\end{array}$ & $\begin{array}{c}\text { Fin de } \\
\text { semana }\end{array}$ & $\begin{array}{c}\text { Vacacional } \\
\text { semanal }\end{array}$ \\
\hline 3-6 años & $4(2,39)$ & $3,93(2,06)$ & $6,24(4,24)$ & $4,55(3,00)$ & $4,1(1,88)$ & $6,68(4,52)$ \\
\hline 7-12 años & $\begin{array}{c}3,95 \\
(2,62)\end{array}$ & $4,41(2,12)$ & $7,02(4,47)$ & $4,63(3,52)$ & $5,60(2,84)$ & $9,09(5,87)$ \\
\hline
\end{tabular}

Tabla 6: Medias de visionado. Fuente: elaboración propia. Se proporciona la Media y el valor de la Desviación típica va entre paréntesis.

Se ha medido si existía una correlación entre las horas de consumo de televisión tradicional entre semana y en fin de semana y se comprobado que existe una correlación positiva con el estadístico de contraste $r$ de Pearson $([N=308]=.605, p<.001)$, de tal manera que aquellos que ven más horas entre semana, también ven más horas en fin de semana. Se da también la misma correlación cuando se analiza el consumo entre semana y fin de semana en el caso de las 0TT $([N=408]=.451, p<.001)$.

También se ha comprobado, utilizando el mismo estadístico de contraste, la relación entre nivel de estudios y consumo de televisión tradicional, existiendo una 
relación negativa significativa en el fin de semana $([N=308]=-.209, p<.001)$. y en vacaciones $([N=308]=-.149, p<.05)$, ya que a mayor nivel de estudios se consumen menos horas de televisión. No se da, sin embargo, ninguna relación estadísticamente significativa entre el nivel de estudios y las horas de consumo de contenidos audiovisuales en plataformas audiovisuales.

Si nos centramos en el tipo de contenidos más consumidos y que forman parte de la "dieta audiovisual" de los más pequeños, se consideró necesario saber qué consumen los niños de entre 3 y 6 años y de entre 7 y 12 años, tanto en la televisión convencional como en las plataformas 0TT siguiendo el análisis comparativo que marcan los objetivos de la investigación.

Los niños/as más pequeños, el contenido que mayoritariamente ven en ambos soportes son series y películas infantiles y lo que menos, las series de ficción. Los padres de los niños/as del segundo intervalo, también declaran que los contenidos más vistos son las series infantiles. Lo niños/as más mayores ven más series de ficción en plataformas que en televisión convencional.

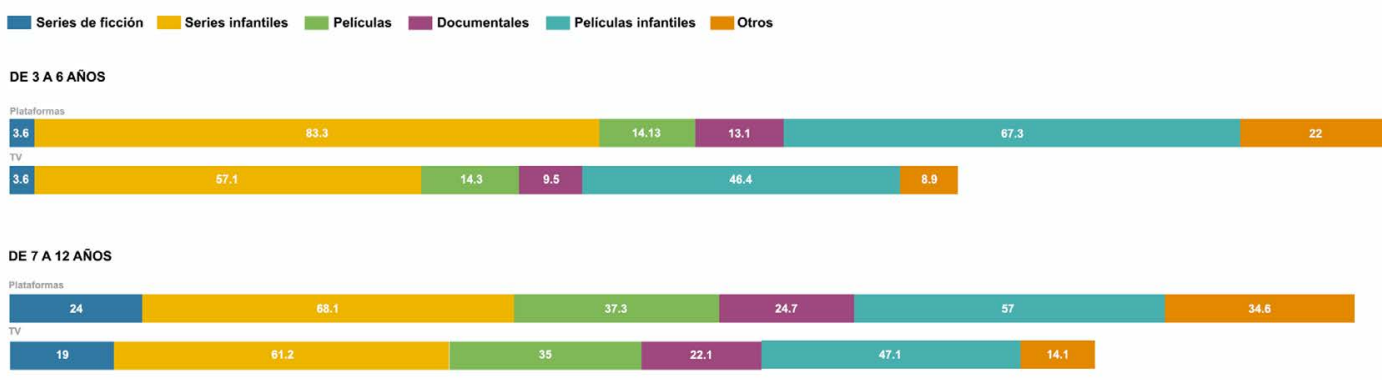

Gráfico 4: Contenidos consumidos. Fuente: elaboración propia.

Cuando se realizó el cuestionario interesaba identificar, además, qué otros contenidos veían los niños de entre 3 y 6 años y de entre 7 y 12 años en plataformas audiovisuales por lo que se programó una respuesta abierta. Así,en el primer intervalo destacan las respuestas de vídeos de YouTube, tanto de canciones, juguetes 0 videojuegos, además de programas y concursos, vídeos de aprendizaje o deportivos. En el segundo intervalo aparecen otro tipo de contenidos como unboxing, youtubers, 
MONOGRÁFICO

videojuegos, vídeos sobre ciencia y experimentos, sobre dibujo e ideas para diseñar $\mathrm{y}$ crear, sobre contenidos graciosos $\mathrm{y}$, finalmente, juegos offline.

Se analizó mediante la prueba $\mathrm{T}$ de Student para muestras independientes si existía alguna relación entre el nivel de estudios de los padres y los contenidos que consumen en la televisión tradicional. No se encontró ninguna relación estadísticamente significativa excepto en la variable películas de tal manera que los hijos de padres con menor nivel de estudio consumen más películas en la televisión tradicional $([N=431]=.066, p<.05)$.

Del total de la muestra se correlacionaron los contenidos que los niños consumían en televisión tradicional y en plataformas audiovisuales. Así, se hallaron resultados estadísticamente significativos en los siguientes casos: aquellos que consumen documentales en televisión tradicional, también consumen series infantiles ( $\chi 21[\mathrm{~N}=431$ ] $=8.818, \mathrm{p}<.005)$, películas $(\chi 21[N=431]=30.139, p<.001)$, series de ficción $(\chi 2$ $1[\mathrm{~N}=431]=4.594, \mathrm{p}<.05)$, películas infantiles $(\chi 21[\mathrm{~N}=431]=6.589, \mathrm{p}<.05) \mathrm{y}$ documentales $\left(\chi^{2} 1[\mathrm{~N}=431]=124.493, \mathrm{p}<.001\right)$ en plataformas audiovisuales. Por su parte, aquellos que consumen películas en televisión tradicional también consumen series $(\chi 21[\mathrm{~N}=431]=23.678, \mathrm{p}<.001)$, películas $(\chi 21[\mathrm{~N}=431]=71.872, \mathrm{p}<.001)$ y documentales $\left(\chi^{2} 1[\mathrm{~N}=431]=13.511, \mathrm{p}<.001\right)$ en plataformas audiovisuales.

Los niños que ven series infantiles en la televisión tradicional también consumen series infantiles $(\chi 21[\mathrm{~N}=431]=23,781, \mathrm{p}<.001)$, películas infantiles $\left(\chi^{2} 1[\mathrm{~N}=\right.$ $431]=13.387, \mathrm{p}<.001)$, series $(\chi 21[\mathrm{~N}=431]=95,663, \mathrm{p}<.001)$, películas $(\chi 21$ $[\mathrm{N}=431]=26,373, \mathrm{p}<.001)$ y documentales $(\chi 21[\mathrm{~N}=431]=5,712, \mathrm{p}<.05)$ en plataformas audiovisuales. Aquellos que ven más películas infantiles en televisión tradicional también ven más series infantiles $(\chi 21[\mathrm{~N}=431]=8,818, \mathrm{p}<.005)$ y películas infantiles $(\chi 21[\mathrm{~N}=431]=30,139, \mathrm{p}<.001)$ en plataformas audiovisuales.

$\mathrm{Si}$ analizamos el número total de tipos de contenido que se consumen en televisión tradicional y en plataformas 0TT también encontramos una relación significativa. Así, a más variedad de contenidos consumidos en televisión, más variedad consumida también en plataformas 0TT $([\mathrm{N}=431]=.293, \mathrm{p}<.001)$. 


\section{Discusión y conclusiones}

La investigación evidencia el uso, cada vez más creciente, de las plataformas audiovisuales para el consumo de los más pequeños, sin embargo, pone de manifiesto que aún no han conseguido desbancar a la televisión tradicional en cuanto al número de horas de consumo entre los niños/as españoles de 3 a 12 años. En cuanto a los escenarios en los que se produce el consumo, las posibilidades que los niños/ as tienen de acceso a las plataformas online, se ven incrementadas con el número de dispositivos conectados a Internet que ya tienen gran parte de los hogares españoles. La televisión ha dejado de verse sólo en la televisión y el teléfono móvil es la estrella de la distribución de contenidos audiovisuales, también en los intervalos de menor edad. Es interesante el dato de que el 70\% declara que sus hijos/as entre 7 y 12 años ya disponen de un dispositivo propio ya que el 19\% de ellos usan su propio teléfono móvil. El acceso que los niños/as tienen a dichas plataformas de contenidos es diverso, aunque queda demostrado, que a mayor nivel de estudios de los padres se diversifican más en los hogares las formas de consumo, pudiendo acceder a los contenidos tanto a través de suscripción, alquiler o compra, descargas o con acceso gratuito. Según los datos de este estudio, el consumo audiovisual se produce en soledad en mayor medida según el niño/a se hace mayor -los niños/as entre 3 y 6 años consumen en un 4,2\% de las veces los contenidos solos frente al $8 \%$ de los que tienen entre 7 y 12 años. La respuesta mayoritaria ha sido la de "a veces", siendo significativa -con un 70,3\% de respuestas- en la franja de edad que recoge a los niños/as más mayores-. A medida que el niño se hace mayor la libertad de este aumenta pudiendo ver contenidos audiovisuales solos, por tanto, sin que se realice una mediación parental que ayude a entender a estos niños/as qué están viendo. Estos datos corroboran que se sigue manteniendo la pauta apuntada por otros investigadores como Aguaded y Díaz-Gómez (2008) y Romero y Monedero (2008), entre otros, que indicaban que en la mayoría de los casos los niños/as ven la televisión sin intervención de un adulto.

En cuanto a lo que consumen, otro aspecto muy importante a analizar, aquellos que ven películas en la televisión tradicional también las consumen en plataformas OTT, aunque en estas, al tener mayor oferta, lo consumido se diversifica añadiendo series y documentales a sus prácticas de visionado. Análogo caso se da con aquellos 
MONOGRÁFICO

que consumen series infantiles en la televisión tradicional ya que añaden a estas series y películas infantiles a su dieta televisiva en plataformas. Por tanto, se da la paradoja de que a mayor oferta en cuanto a contenidos mayor diversidad en cuanto a productos seleccionados para ser vistos.

Se ha detectado una interesante disonancia entre lo más visto y lo que se considera más adecuado. Así, YouTube es la más vista, sin embargo es considerada la menos apropiada por los padres/tutores que consideran la más adecuada Clan TV a pesar de no ser la más vista por lo que se sigue produciendo lo señalado por Vílchez-Martín (1999) quien indica que no se da una conexión entre lo que los niños ven y lo que deberían ver. Este resultado es relevante si se conecta con el modo en que realizan el consumo la mayoría de los niños, en soledad, lo que les permite ver una serie de contenidos que los padres/tutores no consideran adecuados. Además, no solo se produce un consumo sin mediación parental o realizando una mediación parental cómoda (Vílchez-Martín, 1999), sino que también son escasas las regulaciones que se producen sobre cuánto y qué consumir. Se establece en la mayoría de los casos regulaciones de tipo temporal o de contenido (Barrios, 1992, Aguaded, 1999) y no tienen instalado ningún tipo de control parental, especialmente en los más pequeños,quizás porque no suelen ver los contenidos audiovisuales solos, por lo que se puede decir que las familias de este estudio realizan una mediación de tipo restrictiva y en menor medida deslocalizada, pero no evaluativa (Abelman y Pettey, 1989) ya que un 50\% de los padres/tutores de los niños/as de entre 3 y 7 años realizan siempre un visionado previo frente al $49 \%$ de los padres/tutores de los niños/as de entre 7 y 12 años que lo realizan a veces. Sí que habría que destacar que a más nivel de estudios de los padres/tutores, mayor es la regulación sobre el consumo, pues se informan en mayor medida bien en redes sociales, en el colegio o en el grupo de iguales. También a mayor nivel de estudios la oferta consumida es más diversa en cuanto a formatos y temáticas. Aunque también se ha detectado, independientemente del nivel de estudios de los progenitores, que a medida que el niño cumple años el contenido es menos revisado previamente por parte de los padres antes del visionado.

No hay mucha diferencia en cuanto a las horas que consumen los niños de entre 3 y 6 años, entre semana en televisión convencional y en plataformas, lo que supo- 
ne que estos niños ven casi dos horas diarias de contenidos audiovisuales en ambos soportes; media que se mantiene los fines de semana -aunque esto supone que se dobla el consumo diario- y sube exponencialmente en periodos vacacionales. Los cifras son más altas -excepto entre semana en televisión convencional- entre los niños de 7 a 12 años, llegando a consumir una media de nueve horas semanales en periodos vacacionales. Se da, además, un relación perversa ya que los niños/as que más ven la televisión entre semana, independientemente del soporte en el que hagan el visionado, consumen más los fines de semana. Además, habría que tener en cuenta que son los hijos cuyos padres tienen un menor nivel de estudios los que más horas pasan frente al televisor.

Los datos recabados en el estudio que se presenta son convergentes con el estudios precedentes (López Romero y Monedero Morales, 2008; Torrecillas-Lacave, 2013; Hiniker et al., 2016; Solomon-Moore et al., 2018; entre otros.) en cuanto a contenidos, usos y horas de consumo. Además, no se ha detectado que haya habido cambios de comportamiento de los padres/tutores de la televisión tradicional a las plataformas audiovisuales excepto en la mayor cantidad de contenidos diversos consumidos. Los padres/tutores siguen realizando una mediación, en mayor medida, restringida y desenfocada (Van der Voort, Nikken y Van Lil, 1992) o controladoras-restrictivas, permisivas (Llopis, 2004). Apenas se realiza una mediación efectiva y/o didáctica (Vílchez-Martín, 1999), siendo mayormente la mediación efectiva realizada de tipo autocontrol (Torrecillas-Lacave, 2013), más típica de las clases medias-altas que dejan que sea el niño quien decida qué ver y cuando ver, y de tipo heterocontrol (Torrecillas-Lacave, 2013), la predominante en la actualidad y que se limita al establecimiento por parte de los padres/tutores de una serie de normas sobre el uso y consumo. Además, se ha detectado que el uso que se hace de los contenidos podría enmarcarse en lo que Pintado (1998) denominada TV-«está ahí» e incluso en algunos como "canguro catódico" (Urra, 1998).

En este escenario se hace fundamental incidir en los colegios y/o instituciones educativas en la importancia que tiene la alfabetización mediática que dota a los niños/as de las herramientas y estrategias necesarias para saber "leer" contenidos audiovisuales, además de formar a consumidores críticos y maduros en una sociedad cada vez más mediatizada. 


\section{Referencias}

Abelman, R. and Pettey G. (1989). Child attributes as determinants of parental television-viewing mediation. Journal of Family Issues, 10 (2), pp. 251-266. Aguaded, J. I. (1995). La educación en Medios de Comunicación, más allá de la transversalidad. Comunicar, 4, pp. 111-113.

Aguaded, J. I. (1997). La televisión en el nuevo diseño curricular español. Comunicar, 8, pp. 97- 110.

Aguaded, J. I. (1999). Convivir con la televisión. Familia, educación y recepción televisiva. Barcelona: Paidós.

Aguaded, J. I. (2005). Enseñar a ver la televisión: una apuesta necesaria y posible. Comunicar, 25, 1, pp. 51-55.

Aguaded, J. I. y Díaz-Gómez, R. (2008). La formación de telespectadores críticos en educación secundaria. Revista Latina de Comunicación Social, 63, pp. 121139.

Aparici, R. (1994). Televisión, currículo y familia. Madrid: Ministerio de Ciencia y Tecnología.

Austin, E. W. (2001). Effects of family communication on children's interpretation of television. En J. Bryant y J. A. Bryant (Eds). Television and the American Family. Mahwah: Lawrence Erlbaum Associates, pp. 377-395.

Barrios, L. (1992). Familia y televisión. Caracas: Monte Ávila Editores.

Del Río, P., Álvarez, A. y Del Río, A. (2004). Pigmalión. Informe sobre el impacto de la televisión en la infancia. Madrid: Fundación infancia y aprendizaje.

Gabelas-Barroso J.A. y Marta-Lazo, C. (2008). Modos de intervención de los conflictos que supone el consumo de pantallas. Revista Latina de Comunicación Social, 63, pp. 238-252, en http://www.ull.es/publicaciones/ latina/_2008/19_25_Zaragoza/Gabelas_y_Marta.html

García Cortázar, M. et al. (1998). El tercero ausente. Investigación empírica sobre el papel de los adultos en la relación entre niños y televisión. Madrid: Estudios de la UNED.

Fernández, C., Domínguez R., Revilla J.C. y Anagnostou, A. (2004). Formas de legitimación de la violencia en televisión. Política y sociedad, 41, 1, pp. 183199. 
Ferrés, J. (1994). Televisión y educación. Barcelona: Editorial Paidós.

Ferrés, J. (2005). La familia frente al televisor: ¿víctima o culpable?. Comunicar 25, pp. 237-242.

Hiniker, A., Suh, H., Cao, S., Kientz, J.A. (2016). Screen time tantrums: How families manage screen media experiences for toddlers and preschoolers. Computer Supported Parenting. http://faculty.washington.edu/alexisr/ ScreenTimeTantrums.pdf

Livingstone, S. (2007). Los niños en Europa. Evaluación de los riesgos de Internet. Telos 73, pp. 52-69.

López Romero, L. y Monedero Morales, C. R. (2008). Estudio descriptivo del consumo televisivo en primaria y secundaria. Comunicar, $N^{\circ} 31$, pp. 269-273 Llopis, R. (2004): La mediación familiar del consumo infantil de televisión. Un análisis referido a la sociedad española. Comunicación y Sociedad, 17 (2), pp. 125-147.

Martín-Barbero, J. (1987). De los medios a las mediaciones. Méjico: Gustavo Gili. Núñez-Ladevéze, L. y Pérez-Ornia J.L. (2002). Los gustos de la audiencia infantil y la producción televisiva. El conflicto pragmático de los responsables de la audiencia infantil. REIS, 9, pp. 113-143.

Orozco, G. (1996). Televisión y audiencias. Un enfoque cualitativo. Madrid: Ediciones de la Torre.

Pindado, J. (1997). Escuela y televisión: claves de una relación compleja. Cultura y Educación, 5, pp. 25-35;

Pindado, J. (1998). A propósito de las relaciones familia-televisión. Comunicar, 10, pp. 61-67.

Solomon-Moore, E., Matthews, J., Reid, T., Toumpakari, Z., J. Sebire, S., Thompson, J. L., Lawlor, D.A.y Jago, R. (2018). Examining the challenges posed to parents by the contemporary screen environments of children: a qualitative investigation. Pediatrics , 18: 129 https://doi.org/10.1186/ s12887-018-1106-y

Torrecillas Lacave, T. (2013): Los padres, ante el consumo televisivo de los hijos: Estilos de mediación. Revista Latina de Comunicación Social, 68. http://www. revistalatinacs.org/ 068/paper /968_CEU/02_Torrecillas.html D0I: 10.4185/ RLCS-2013-968 
Urra, J. (1998). Niños y no tan niños. Madrid: Biblioteca Nueva

Van Der Voort, T. H., Nikken, P. y Van Lil, J. P. (1992). Determinants of Parental Guidance of Children's Television Viewing. A Dutch replication study. Journal of Broadcasting and Electronic Media, 36, pp. 61-74.

Vílchez-Marín, L. F. (1999). Televisión y familia. Un reto educativo. Madrid: PPC.

\section{(c)}

Este obra está bajo una licencia de Creative Commons Reconocimiento 4.0 Internacional. 